\title{
Alleviation of Stationary Biases in a GCM through a Mountain Drag Parameterization Scheme and a Simple Representation of Mountain Lift Forces
}

\author{
FRANÇOIS LOTT \\ LMD-UPMC Paris 6, Paris, France
}

(Manuscript received 6 January 1998, in final form 27 May 1998)

\author{
ABSTRACT
}

\begin{abstract}
The problem of the representation of the orientation of mountain forces in a GCM is examined. First a series of winter simulations is presented with the Laboratoire de Météorologie Dynamique GCM where the model ground is flat and where the mountains are represented by applying forces to the flow that are either strictly opposite to the local wind (i.e., a drag) or strictly perpendicular to it (i.e., a lift). These experiments show that the drag forces improve the zonal wind but do not improve the steady planetary wave everywhere, while the lift forces scarcely affect the zonal wind but force a steady planetary wave that is everywhere realistic. The drag force is formulated following the realistic subgrid-scale orographic (SSO) scheme, developed recently at ECMWF. The lift force essentially enhances vortex stretching over large-scale mountains.

To address issues concerning the representation of SSO in GCMs, experiments with mean orography, the ECMWF SSO scheme, and enhanced lift forces over mountain massifs are compared to experiments with mean orography only. They confirm the results of the "no mountain" runs: the SSO scheme improves the zonal wind and the enhanced lift forces improve the steady planetary wave. Although these results suggest that the recent SSO schemes that are validated against data, and that significantly decelerate the low-level flow, are beneficial to GCMs, they also raise the question of the representation of the orientation of mountain forces that should be included in these schemes. For this purpose, the lift representation proposed should be considered carefully in an operational context, because it is only linked to the problem of SSO parameterization by qualitative arguments.
\end{abstract}

\section{Introduction}

Mountains influence the dynamics of the atmosphere at many different length scales. For instance, they force gravity waves that take angular momentum from the earth and transport it through the atmosphere over long distances (Queney 1947; Lilly et al. 1982). At low levels, mountains exert a drag on the large-scale flow through nonlinear processes that are now well established (Hunt and Snyder 1980; Smolarkiewicz and Rotunno 1989; Schär and Smith 1993). At large scales, mountains contribute to the steady planetary wave (Hoskins and Karoly 1981; Valdes and Hoskins 1989), to the storm tracks (Hoskins and Valdes 1990), and to the lowfrequency variability (Charney and De Vore 1979; Marcus et al. 1994). Accordingly, in general circulation models (GCMs), it seems natural to try to correct the model errors on these steady and quasi-steady structures by improving the representation of the forces exerted on the flow by mountains. For instance, the parameterization of mountain gravity waves alleviates systematic

Corresponding author address: Dr. François Lott, LMD-UPMC Paris 6, Case Courrier 99, 4 Place Jussieu, 75252 Paris Cedex 05, France.

E-mail: flott@1md.jussieu.fr errors in GCM simulations of the tropospheric westerlies (Palmer et al. 1986). Miller et al. (1989) extended this study, showing that the representation of orography in global models has an impact (positive or negative) on many of the large-scale structures mentioned before. Miller et al. (1989) and also Stephenson (1994) have shown that the vertical repartition of mountain drag is an important issue for GCMs. These large-scale simulations obviously provide important applications for the recent 2D (Clark and Peltier 1984; Durran and Klemp 1987; Scinocca and Peltier 1994) and 3D (Miranda and James 1992; Schär and Durran 1997) theoretical studies on mountain flow. Following these, Lott and Miller (1997, hereafter LM97) have proposed a subgrid-scale orographic (SSO) drag scheme that gives particular attention to the drag of the flow at levels that intersect the subgrid-scale orography. This scheme is also validated against the Pyrenees Experiment data (PYREX) (Bougeault et al. 1993) in all the situations for which the incident wind perpendicular to the ridge is strong.

Among the quasi-steady patterns that SSO parameterization schemes affect, the steady planetary wave is of particular interest because its simulation is rather difficult. For instance, Fig. A1 in D'Andrea et al. (1998) shows the steady geopotential height field for 15 different models: among them, many (but not all!) are too 
180

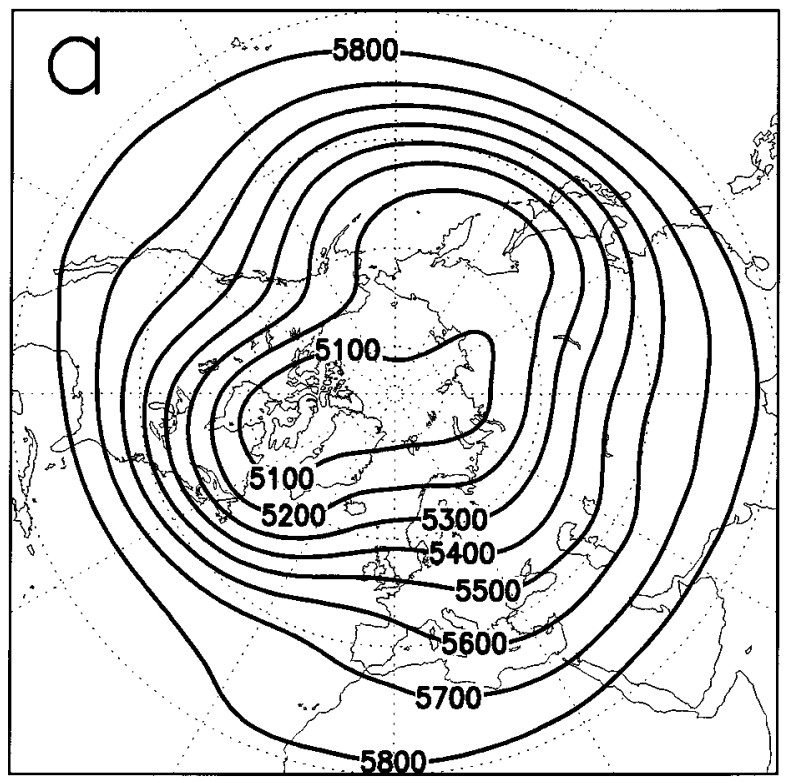

0

180

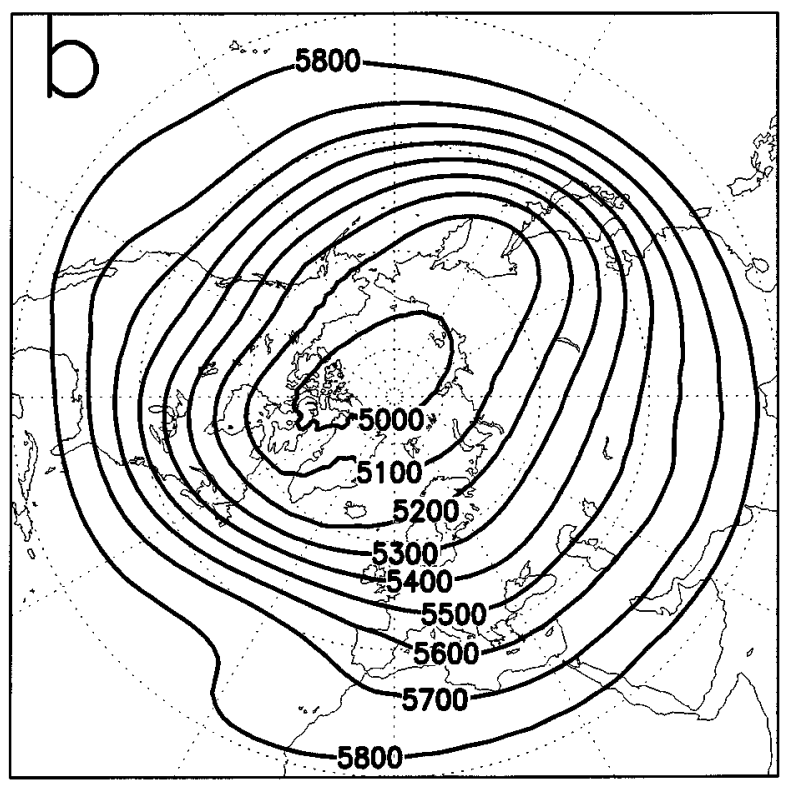

0

FIG. 1. The 500-hPa geopotential height averaged over the winter months (Dec-Jan-Feb, DJF) for the period 1985-93: (a) NMC analysis; (b) LMD control $96 \times 72 \times 19$.

zonal. This type of error is illustrated in Fig. 1, which shows that in the GCM used in this paper, the ridges over the Rockies and the Himalayan Plateau are not strong enough when compared to the National Meteorological Center (NMC, now known as the National Centers for Environmental Prediction) analysis. To cor- rect these errors one has to determine which processes related to the mountains contribute to the steady planetary wave. For this purpose the quasigeostrophic (QG) models are helpful because they have been extensively used in diagnosing the forcing of planetary waves [Charney and Eliassen (1949); a review is also given in Held (1983)]. In these models, mountains induce vortex stretching, modifying the vorticity but keeping the potential vorticity unchanged. It is noteworthy that these models have also been used extensively to study atmospheric low-frequency variability and blocking (Charney and Strauss 1980). Interestingly, the circulation over mountains associated with vortex stretching is driven by a force whose horizontal component is perpendicular to the incident flow in the linear quasigeostrophic context and that is proportional to the mountain volume (Smith 1979). In the context of atmospheric models that do not use the quasigeostrophic approximation, the fact that vortex stretching can be eventually accounted for by a force that is quite predictible is potentially promising: it can be easily parameterized and tuned, without changing the model lowest-level elevation; it can help reduce model errors on the representation of the mountain force component that is perpendicular to the incident wind (Lott 1995); and it can help reduce errors on the model simulation of the planetary waves.

A basic asumption of the present study is that the steady planetary waves are largely driven by the mountains. Although this view is partly supported by linear studies of the atmospheric responses to thermal and orographic forcing, it is well known that thermal forcings are also very important (Valdes and Hoskins 1989). This is particularly evident over the Pacific and North America, where the tropical sea surface temperature and precipitation fluctuations associated with the Southern Oscillation modify very significantly the seasonal mean planetary waves (Horel and Wallace 1981). This tropical-extratropical connection can be understood in terms of Rossby waves propagating from the Tropics toward the midlatitudes (Hoskins and Karoly 1981).

On the basis of GCM simulations, the first purpose of the paper is to suggest that it is important to represent correctly the orientation of the mountain forces if one wishes to simulate properly the extratropical climate. The second purpose of this paper is to show that the introduction of lift forces does not modify the beneficial impact of conventional SSO drag schemes on the zonal mean flow. The last purpose of the paper is to show that the LM97 SSO scheme, which treats the SSO mountain drag forces rather differently than in the past, can be beneficial for long GCM runs. Nevertheless, the purpose of the paper is not to propose an operational form for lift parameterization. Indeed, the lift representation adopted has been chosen to be as simple as possible, in order not to hide the fact that the way mountains exert sideways forces on the atmosphere is better understood for large-scale mountains then for SSO. Furthermore, 
the arguments given in section $2 \mathrm{a}$ that could justify the use of this lift representation in the context of SSO parameterization are only qualitative.

In section 2, the parameterization schemes used are presented and a brief discussion is also given of their impact on the large-scale flow, in the quasigeostrophic context. In section 3, GCM tests are made. First, fivewinter integrations are presented where the orography in the model is suppressed (the lower boundary is set everywhere to the sea level) and where the mountains are entirely parameterized either by large low-level drag or by large low-level lift. Second, a 10-winter run is made where mean orography is used in the model as a lower boundary and where the parameterization of the mountain forces is used as a correction that should improve the model. In section 4 the implications of the results obtained are discussed. The appendix gives further justifications for some modifications of the SSO drag schemes by comparison with LM97.

\section{The different parameterizations}

\section{a. General considerations}

In many cases in fluid dynamics the force exerted by an obstacle on a fluid is oriented in a direction that is different from that of the incident wind. The drag is the component of the force $\mathbf{F}$ that decelerates the fluid because it is opposite to the wind and works against it,

$$
\mathbf{u} \cdot \mathbf{F}<0 .
$$

In nature, the low-level wake behind three-dimensional mesoscale high obstacles can be understood as the result of the drag the obstacle exerts on the flow at the levels that do not pass over the orography. Since this force is related to mountains whose horizontal scale is far beyond that of a GCM grid box, it needs to be parameterized. A way to do this is to apply drag forces directly into the flow at the model levels that are believed to intersect the subgrid-scale orography (LM97). Since at the same horizontal scales, small mountains emit gravity waves, an SSO scheme has to deal with blocked flow drag and gravity waves altogether and in a fraction that depends on the incident flow characteristics and on the SSO height. In the following, the forces related to these mesoscale processes will be referred to as drag forces, which is a shortcoming since the gravity wave's force, for instance, can be oriented in a direction that differs from that of the wind, because the former turns with height and/or because of the anisotropy of the ridge (Phillips 1984). These effects are partly taken into account in LM97 but the numerical experiments carried out in the context of this work have shown that this directional issue is not essential. This is related to the scheme formulation itself, where the critical levels are not treated as they should be in the 3D context (Shutts 1995; Broad 1995), and because the U.S. Navy dataset, used to evaluate the mountains characteristics, is too coarse and probably underestimates the anisotropy of the ridges. Rather similar directional effects probably occur for the force an anisotropic mountain induces on the low-level flow. Since these effects are not often discussed in the literature, they are not included in the blocked flow part of the LM97 scheme, where only forces opposite to the local wind are parametrized.

The lift is the component of the force $\mathbf{F}$ that modifies the direction of the flow but does not decelerate it and does not work against it. A good example of pure lift in geophysical fluid dynamics occurs in quasigeostrophic flows over low ridges, during the process of vortex compression. As often assumed in a GCM, this force is correctly represented when the model lower boundary is the mean orography, the lift being essentially related to the mountain volume. Nevertheless, some models use enhanced orography (envelope) essentially because the planetary wave response to orographic forcing is too small. This orography enhancement is based on the fact that between the narrow ridges of a mountain massif air can be blocked locally, and can be dynamically separated from the large-scale flow, if the valleys are not well ventilated. In this case, a region of complex terrain probably acts as if it has a larger than actual height, and a mean orography underestimates the lift. In this context, as local air blocking in valleys occur for mountains with large slope, and because these effects are still not handled well in the boundary layer schemes, the enhanced lift can be viewed as an SSO parameterization at scales smaller then those accounted for in the LM97 scheme.

As this discussion shows, there are many processes that make the orientation of the mountain forces different from that of the wind. Only a few are well documented and even fewer are properly parameterized. A way to assess if this directional issue is important is to carry out GCM experiments modulating the orientation of the mountain forces. In the following, this will be done using the LM97 SSO scheme for the drag component and a representation of the lift that follows Smith (1979). This approach ensures that the amplitude of each mountain force components is quite realistic.

\section{b. Subgrid-scale orographic drag}

The orographic drag scheme used in the present study is the one presented in LM97. For clarity and convenience a brief description is given here with emphasis on a few modifications undertaken to adapt the scheme to a low-resolution GCM. The SSO over one gridpoint region (GPR) is represented by seven parameters, $\mu, \gamma$, $\sigma, \theta, Z_{\min }, Z_{\max }$, and $Z_{\text {mea }}$, which stand for the standard deviation, the anisotropy, the slope, the orientation, the minimum, the maximum, and the mean elevation of the orography, respectively. These seven parameters are evaluated over a GPR from the U.S. Navy $\left(10^{\prime} \times 10^{\prime}\right)$ dataset. The scheme uses values of low-level wind and static stability, which are partitioned into two parts. The 
first corresponds to the incident flow and is evaluated by averaging the wind, the Brunt-Väisälä frequency, and the fluid density between the model ground $Z_{o}$ and the mountain peaks $Z_{\max }$ (in the simulations that follow $Z_{o}$ will be either 0 or the mean orography, $Z_{\text {mea }}$ ). This low-level flow is referenced as $\mathbf{U}_{H}, N_{H}$, and $\rho_{H}$, respectively. The second part is the "blocked" flow, whose upper height $Z_{b}$ is the highest level that satisfies the condition

$$
\int_{Z_{b}}^{Z_{\max }} \frac{N}{U_{p}} d z \leq H_{\mathrm{NC}}
$$

where the wind speed $U_{p}(z)$ is calculated by resolving the wind $\mathbf{U}(\mathbf{z})$ in the direction of the incident flow $\mathbf{U}_{H}$. The parameter $H_{\mathrm{NC}}$ tunes the depth of the blocked flow layer and is of order one. Then, for each layer below $Z_{b}$ a force per unit volume is applied:

$$
\begin{aligned}
\mathbf{D}_{b}(z)= & -\rho C_{d} \max \left(2-\frac{1}{r}, 0\right) \frac{\sigma}{2 \mu}\left(\frac{Z_{b}-z}{Z_{b}-Z_{o}}\right) \\
& \times\left(B \cos ^{2} \psi+C \sin ^{2} \psi\right) \frac{\mathbf{U}|\mathbf{U}|}{2}
\end{aligned}
$$

In Eq. (2) the angle between the incident flow and the normal ridge direction is $\psi$, the aspect ratio of the obstacle as seen by the incident flow is $r$, and the constants $B(\gamma)$ and $C(\gamma)$ are as in LM97. The parameter $C_{d}$ tunes the blocked flow drag amplitude. Following the literature on flow dynamics around a bluff body, $C_{d}$ is of order 1 .

On the supposition that when there is low-level flow blocking, the effective height of the SSO seen by the fluid is reduced to $Z_{\max }-Z_{b}$, the gravity wave stress (i.e., force per unit area) is reduced accordingly. It becomes after evaluation of the typical number of ridges within a GPR

$$
\begin{aligned}
\tau= & \rho_{H} G U_{H} N_{H}\left(Z_{\max }-Z_{b}\right)^{2} \frac{\sigma}{4 \mu} \\
& \times\left(B \cos ^{2} \psi_{H}+C \sin ^{2} \psi_{H} ;(B-C) \sin \psi_{H} \cos \psi_{H}\right) .
\end{aligned}
$$

The parameter $G$ tunes the gravity waves stress amplitude and is also of order 1 . The vertical partition of the gravity wave stress determines the levels at which the waves are dissipated and slow down the mean flow. First, the stress decays by a tunable factor $\beta<1$, between the ground and the $850-\mathrm{hPa}$ level to crudely account for the low-level dissipation of the trapped lee waves [see Miller et al. (1989) and the appendix]. Above, breaking occurs when the total Richardson number, $\mathrm{Ri}$, falls below a critical value $\mathrm{Ri}_{c}$, which is of order unity.

\section{c. Orographic lift}

In the model itself, the lift representation consists of applying a force per unit volume $\mathbf{L}$ that is perpendicular to the wind at each levels below the mountain maximum height $Z_{\max }$ :

$$
\mathbf{L}=-\rho C_{l} f\left(\frac{Z_{\max }-Z}{Z_{\max }-Z_{o}}\right) \mathbf{k} \times \mathbf{u} .
$$

In Eq. (4), $f$ is the Coriolis frequency and $C_{l}$ is a tunable parameter of order 1 . When the incident wind is uniform in the vertical, Eq. (4) integrated from the model ground $Z_{o}$ to the mountain peak $Z_{\max }$ gives a stress,

$$
\mathcal{L}=-\rho C_{l} f\left(\frac{Z_{\max }-Z_{o}}{2}\right) \mathbf{k} \times \mathbf{u},
$$

which is similar to the lift stress exerted by an obstacle of mean height $\bar{h}$ on a quasigeostrophic flow (Smith 1979)

$$
S=-\rho f \bar{h} \mathbf{k} \times \mathbf{u},
$$

provided that the maximum mountain elevation is twice the mountain mean when $Z_{0}=0$ and $C_{l}=1$. An upper bound for this enhancement is obtained by taking $C_{l}=$ 2 , which means that the large-scale flow passes over the mountain peaks and is compressed by an amount equal to the volume of the atmosphere located below these peaks. In the following the intermediate value $C_{l}=1$ will be adopted.

In the context of SSO parameterization, the arguments given in section $2 \mathrm{a}$ to enhance the lift over large-scale mountains are very similar to those used to introduce envelope orographies is some large-scale models (Wallace et al. 1983). Nevertheless, in the context of the present work the lift representation is better adapted, since envelope orography increases all the components of the mountain-induced forces in a ratio that is not easy to control. In other contexts, it is not the intention of this paper to suggest that one should replace envelope orographies in the GCMs that use them with the simple lift representation given here. Indeed, to impose lift forces in the flow at well-defined model levels is in many respects very different from what occurs in the process of vortex compression where the lift force is also transmitted vertically: at each level above the obstacle, the isentropes are distorted and the volume of all the isentropes deformation equals the mountain volume. In this case, the sideways force goes with a vertical uplift, which cannot be parameterized easily in an hydrostatic model, except by changing the lower boundary height.

\section{d. Impact in a shallow-water quasigeostrophic flow}

To adress some issues about the response of the largescale flow to momentum forcings close to those presented before, we consider a shallow-water flow of depth $H$, which is distorted by the force $\mathbf{F}$ or/and by an explicit mountain $h$. For this fluid the equations of motion can be written 


$$
\left\{\begin{array}{l}
d_{t} u-f v+\frac{1}{\rho} \partial_{x} p=\frac{1}{\rho} F_{x} \\
d_{t} v-f u+\frac{1}{\rho} \partial_{y} p=\frac{1}{\rho} F_{y} \\
d_{t} H=H \boldsymbol{\nabla} \cdot \mathbf{u}=0,
\end{array}\right.
$$

and we assume an incident flow $U(y)$ driven by a sloping free interface of elevation $\nu=\nu_{0}$ above a reference flow depth $H_{0}$,

$$
H(y)+H_{0}=\nu_{0}(y)=H_{0}-\int_{0}^{y} \frac{f}{g} U(s) d s .
$$

Under the action of the force $\mathbf{F}$ and/or of the mountain $h$, the fluid response can be described by the potential vorticity equation

$$
\frac{d}{d t} \frac{\xi+f}{H_{0}+\nu-h}=\frac{\boldsymbol{\nabla} \times \mathbf{F}}{\rho\left(H_{0}+\nu-h\right)} .
$$

For large-scale and small-amplitude forcings, $F$ and $h$, the quasigeostrophic version of the potential vorticity equation can be adopted:

$$
\frac{d}{d t}\left(\Delta \Psi-\frac{f_{0}^{2}}{g H_{0}} \Psi+f+f_{0} \frac{h}{H_{0}}\right)=\frac{1}{\rho} \boldsymbol{\nabla} \times \mathbf{F},
$$

where $\Psi$ is the streamfunction. Now, if a flow force that is everywhere perpendicular to the wind, $\mathbf{F}=$ $-\rho e(x, y, \mathbf{u}) \mathbf{k} \times \mathbf{u}$, is introduced, it can be included in the potential vorticity equation, leading to

$$
\frac{d}{d t}\left(\Delta \Psi-\frac{f_{0}^{2}}{g H_{0}} \Psi+f+f_{0} \frac{h}{H_{0}}+e(x, y, \mathbf{u})\right)=0 .
$$

One sees that in the QG limit, it is equivalent to specify a lower bound $h$ or a lift forcing, providing that $e=$ $f_{0} h / H_{0}$, an expression that is very close from the lift force (4) averaged vertically over the fluid depth $H_{0}$. In the quasigeostrophic limit, it is important to note that the lift representation used is exactly equivalent to the orography representation used in the many quasigeostrophic studies of the influence of mountains on largescale flow. By integration over $y, z$ of the meridional momentum equation in (7) it is also straightforward to verify that when $e=f_{0} h / H_{0}$, the overall force exerted on the fluid is, to the lowest order in the forcing amplitude, opposite to the mountain lift force that exists on an obstacle $h$ in a large-scale flow (Smith 1979).

In this context one can also determine qualitatively the impact of a drag force like (2) on the large-scale flow. For simplicity we only retain here that the drag force is opposite to the wind:

$$
\mathbf{F}=-\rho e(x, y, \mathbf{u}) \mathbf{u} .
$$

This force modifies the potential vorticity because it induces a potential vorticity flux that is perpendicular to the flow:

$$
\begin{aligned}
& \frac{d}{d t}\left(\Delta \Psi-\frac{f_{0}^{2}}{g H_{0}} \Psi+f+f_{0} \frac{h}{H_{0}}\right) \\
& \quad=\boldsymbol{\nabla} \cdot[e(x, y, \mathbf{u}) \mathbf{k} \times \mathbf{u}] .
\end{aligned}
$$

Accordingly, a drag induces a potential vorticity dipole, downstream of the area where it is applied. When the forcing is related to the parameterization of SSO, this produces a wake downstream of the mountains that is the results of the retardation of the large-scale flow by all the individual mesoscale peaks behind which mesoscale wakes are known to occur (Miranda and James 1992; Schär and Durran 1997). There is some evidences that those large-scale wakes exist (Lott 1995) and that they can be reproduced by low-level drag parameterizations (LM97).

Within a similar approach it is easy to verify that the lift representation induces potential vorticity fluxes that are always along the streamlines. In the steady case, it means that the potential vorticity anomalies induced by the lift remain located over the area where the lift force is applied and never extend downstream of it: when a fluid parcel looses potential vorticity locally, because the vorticity flux diverges, it is advected toward the region where the same vorticity flux converges and where it gains back all the potential vorticity it has lost. This property is already apparent in Eq. (11), the lift being included in the advective part of the potential vorticity equation.

\section{Impact on winter climatic runs}

\section{a. Methodology}

The model used in this study is the Laboratoire de Météorologie Dynamique (LMD) GCM (Sadourny and Laval 1984), which was recently rewritten. Grid points are regularly distributed in the longitude-latitude coordinates with resolution $3.75^{\circ} \times 2.5^{\circ}$ and it has 19 vertical sigma levels unevenly spaced to provide more resolution near the ground and in the lower stratosphere. The horizontal resolution has been adopted to ensure that the synoptic weather systems are resolved well enough to transport the correct amount of angular momentum between the Tropics and the extratropics (Palmer et al. 1986), while keeping the numerical cost of the simulations low. The upper boundary is a rigid lid, which means that the planetary waves that could propagate toward the middle atmosphere are reflected back in the troposphere. This is a defect whose impact on the large-scale flow simulation can be as significant as that of any single physical parameterization. Obviously, the importance of the results obtained hereafter is limited by this defect. For the following discussion it is also important to note that in the boundary layer scheme the roughness length is not increased over mountains. To perform simulations that can be compared to the observed climate, and to ensure that the results presented are significant, the model is integrated over long 
periods and forced with observed SST and sea-ice distributions. The longest experiment lasts $9.5 \mathrm{yr}$ (July 1984-December 1993) starting with initial conditions built from European Centre for Medium-Range Weather Forecasts (ECMWF) analyses. It is the control run, and it is analyzed during the winter months after January 1985 when model equilibration is achieved. In this control run mountains are represented explicitly, $Z_{0}=Z_{\text {mea }}$, and there is no parameterized orographic forces. In the other experiments, the orography representation is modified. In subsection $3 \mathrm{~b}$ are presented three different sets of five-winter runs (January 1985-December 1990) with no explicit mountains $\left(Z_{0}=0\right)$, but with three different mountain force parameterizations (no force, lift only, drag only). In section $3 \mathrm{c}$ are presented one set of ninewinter (January 1985-December 1993) integration with mean orography $\left(Z_{0}=Z_{\text {mea }}\right)$ and with mountain drag parameterization and enhanced lift forces. In these four different sets of experiments each winter starts a month in advance from control run fields to allow model equilibration.

\section{b. Experiment with forces and without explicit mountains}

Experiments without model-resolved orography $\left(Z_{0}\right.$ $=0$ ) and with parameterized forces are performed to verify that the mountains affect the model climate and to determine which forces are important to simulate properly the large-scale flow. In the first experiment there is no parameterized force; in the second a large drag is applied to the flow below $Z_{\max }$. This large drag is provided to the flow using the low-level drag part of the SSO scheme with $H_{\mathrm{NC}}=0, C_{d}=1$, and without gravity wave drag $(G=0)$. In the third experiment, a large lift force is applied between 0 and $Z_{\max }$, using the low-level lift part of the scheme with $C_{l}=1$. The resulting zonal mean flows are shown in Fig. 2 and compared to the NMC analysis (Fig. 2a). (Figure 2b shows that in the absence of any mountain representation, the model has westerlies in the Northern Hemisphere that are too strong at all levels. At each altitudes below 200 $\mathrm{hPa}$ the zonal wind maximum in the model is $5 \mathrm{~m} \mathrm{~s}^{-1}$ too large when compared to the climatology Fig. 2a). Above the tropopause, the jet does not close and reaches values in the stratosphere that are more then $30 \mathrm{~m} \mathrm{~s}^{-1}$ larger than the climatology. When the low-level drag is introduced (Fig. 2c), the errors on both the low-level winds and the jet maximum are significantly reduced. However, the low-level drag does not reduce the errors in the upper levels (above $100 \mathrm{hPa}$ ), which is natural since the gravity wave part of the scheme is not activated in this set of experiments. To a certain extent, the large low-level lift in Fig. 2d also has a beneficial impact on the zonal mean, but it is less pronounced than for the drag. In this case, the impact essentially follows that the time-mean flow is not as zonal as it is without any mountain forcing. It also follows that the lift represen- tation can account for a topographic Rossby wave drag, in agreement with quasigeostrophic models results (Charney and Strauss 1980).

Figure 3 shows the planetary waves in these three sets of experiments. Figure 3 b shows that without orography; the steady planetary wave in the model is very weak compared to the climatology (Fig. 3a). This means that in the model used the land-sea contrast and the associated nonzonal thermal forcings do not contribute significantly to the steady planetary wave. With large low-level drag (Fig. 3c), the model response shows a pronounced planetary wave response, with dominant wavenumber 1 , which is quite realistic over the eastern Atlantic and the western Pacific; it is nevertheless far from the climatology over North America and Asia. With large low-level lift (Fig. 3d) the steady planetary wave has dominant zonal wavenumbers $2-3$ as in the observed climatology. In this case, the model response is quite close to the climatology everywhere (regarding the fact that runs without explicit orography are rather extreme!). Compared to the drag-only run, the most striking improvement is over the Rockies and the Himalayas, where the lift has a direct local effect and simulates the ridges correctly. It is noteworthy that this direct local effect is the most natural one that could be expected from the lift parameterization. Indeed, as shown in section $2 \mathrm{~d}$, the lift parameterization is introduced to replace large-scale air uplift and the associated vortex compression by a force. As the first effect of vortex compression in the midlatitudes is to give rise to anticyclonic circulation over the ridges, it naturally improves the flow there. The fact that improvements eventually appear far from the ridges indicates that the lift also forces realistic Rossby waves, which is also not very surprising since quasigeostrophic models can reproduce those waves realistically and since in quasigeostrophic models lift parameterization and vortex compression are nearly equivalent (section $2 \mathrm{~d}$ ).

\section{c. Experiments with forces and mean orography}

The preceding results suggest that mountain drag forces act primarily to retard the zonal flow, while lift forces act primarily to force a steady planetary wave structure. In a different set of experiments (not presented here) where the different parts of the orography parameterization scheme were each tested independently, it was found that most results obtained without explicit mountains are also valid with mean orography. For instance, the low-level blocked flow drag improves the low-level flow. Nevertheless, it was generally found to degrade the steady planetary wave over the continents and did not affect it much over the oceans, somehow in contradiction with the results obtained without explicit mountains. From a more general point of view, by performing different sets of experiments where the parameters of the SSO drag scheme were changed, it was found difficult to improve significantly the steady 

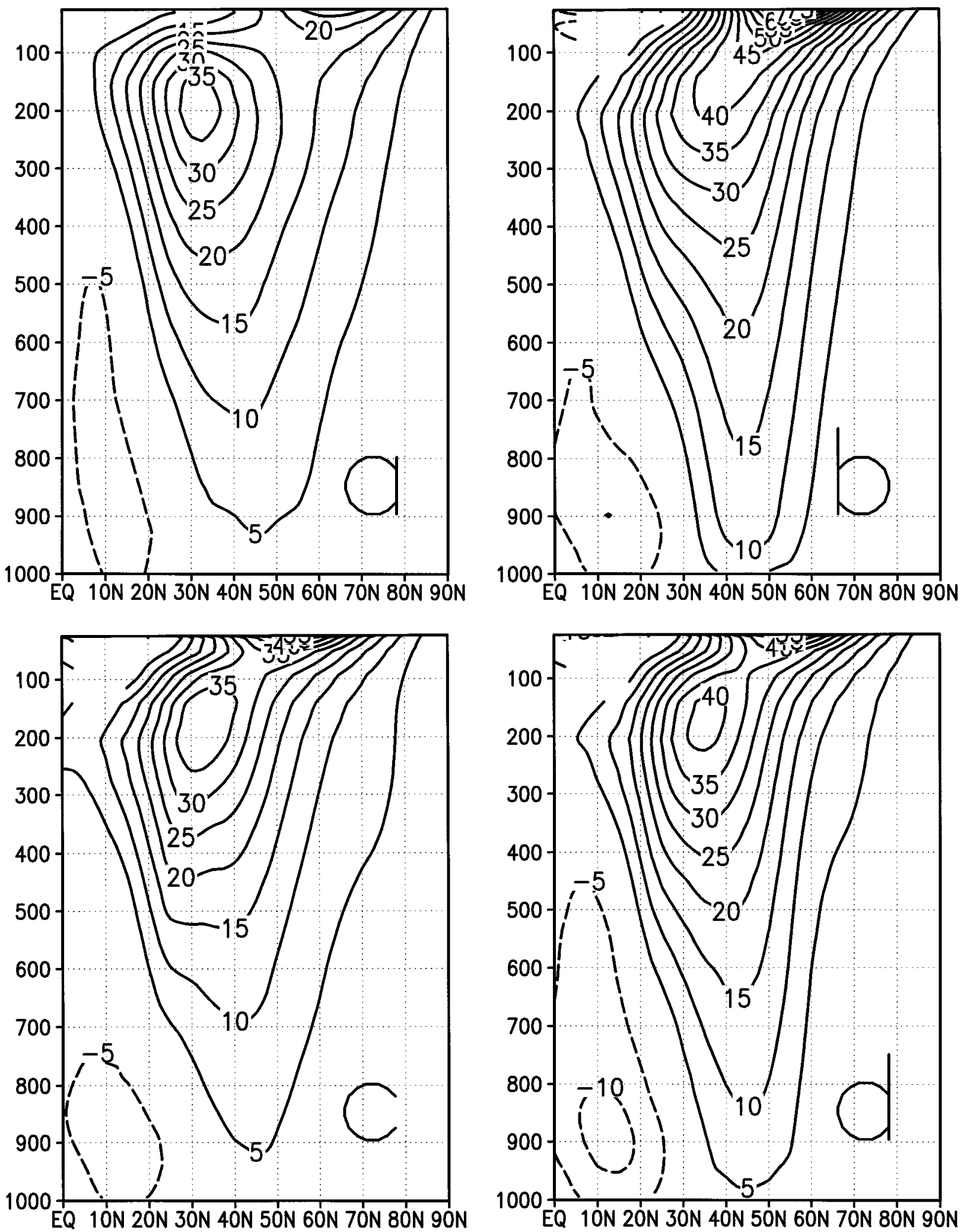

FIG. 2. Zonal-mean wind averaged over the winter months (DJF) of the period 1985-90. LMD run with no explicit orography. (a) NMC analysis; (b) LMD no drag, no lift; (c) LMD low drag only; (d) LMD low lift only. Zero line not shown; negative values are dashed. 
180

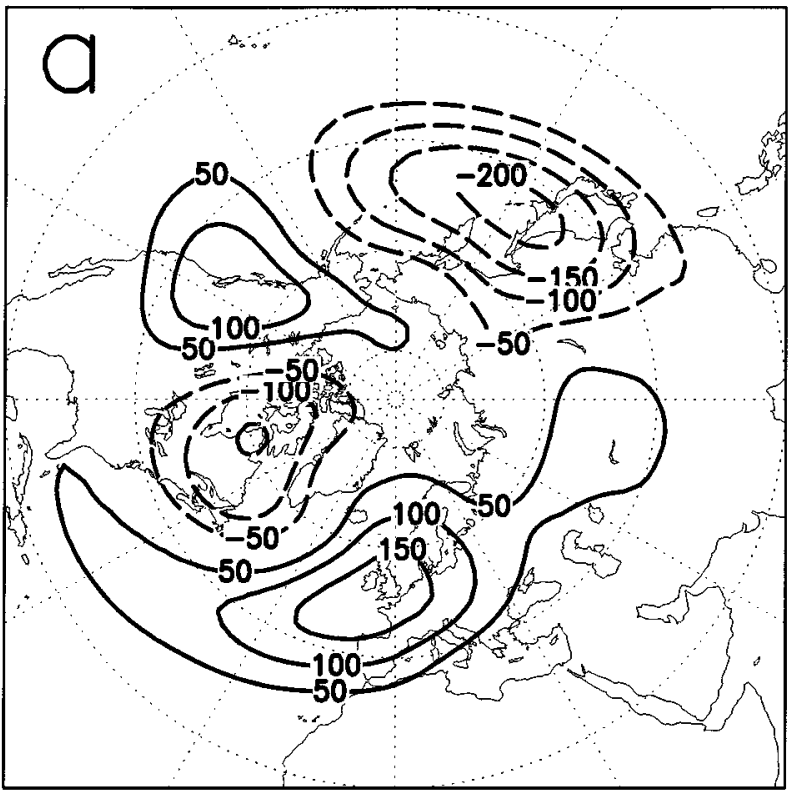

0

180

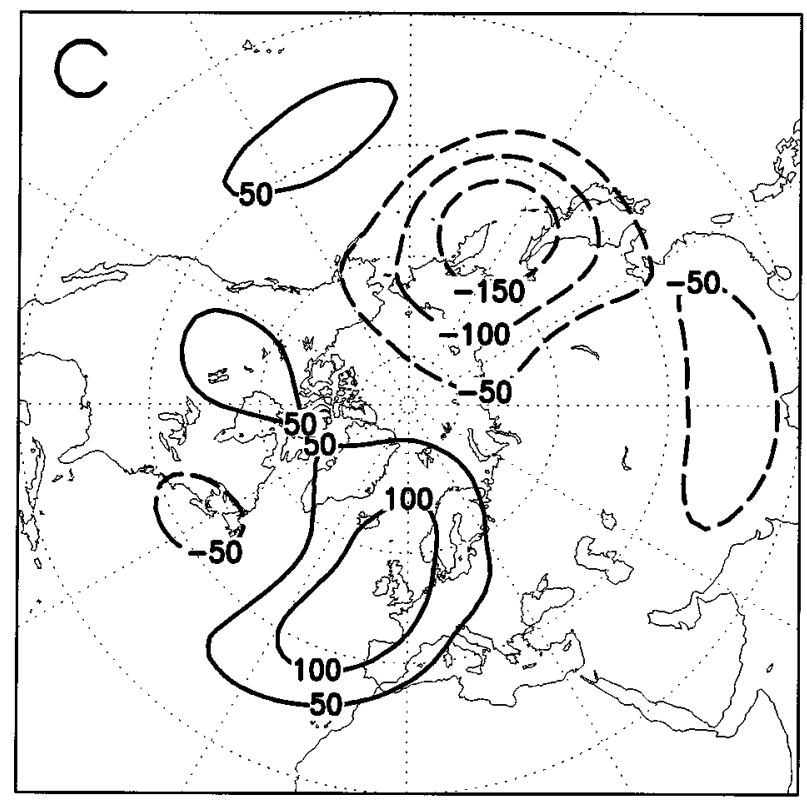

0
180

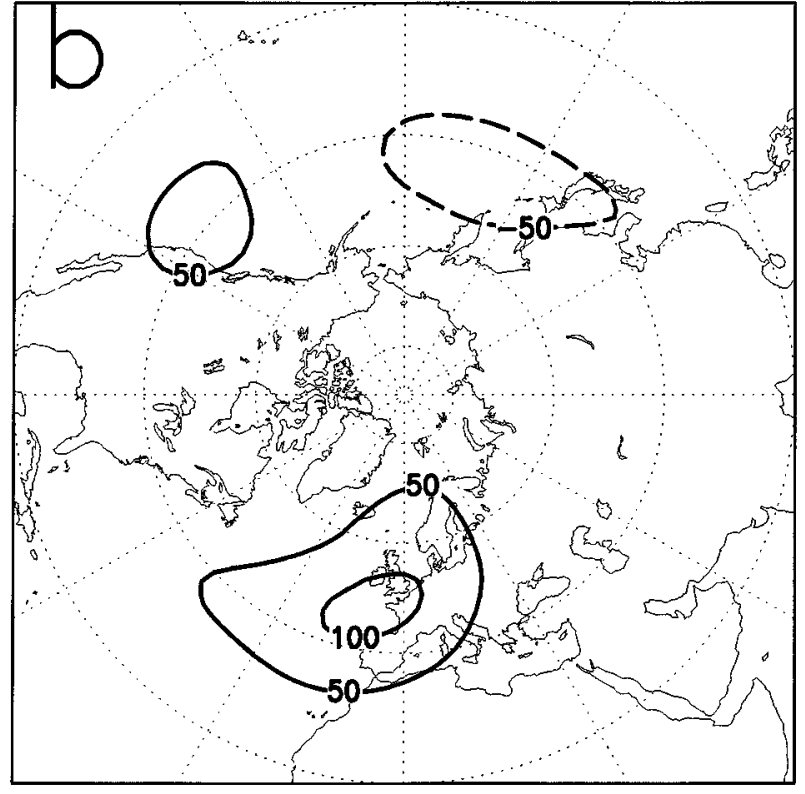

0

180

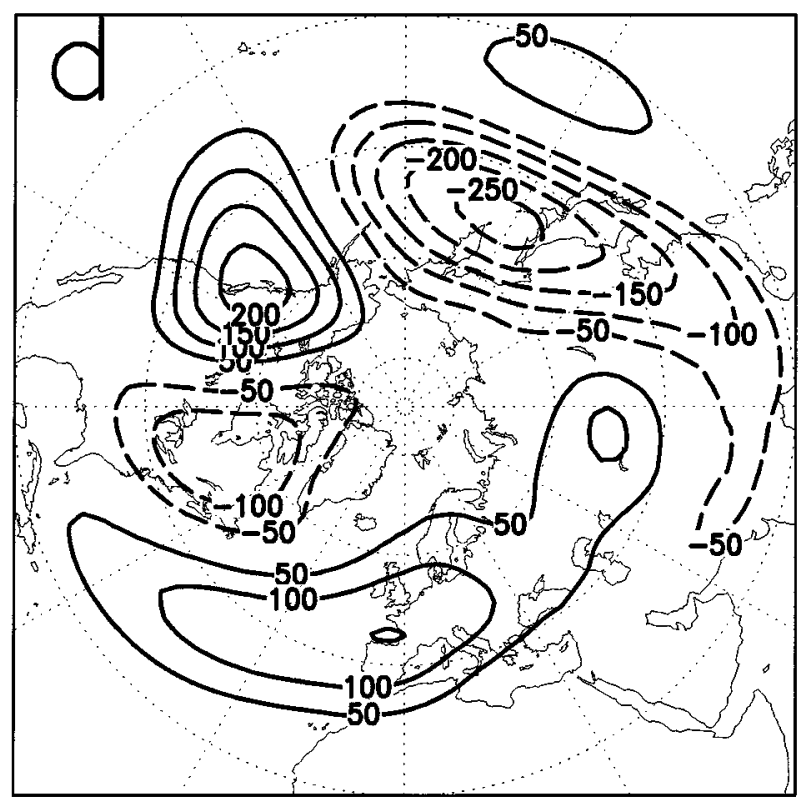

0

FIG. 3. Anomaly to the zonal mean of the geopotential height at $500 \mathrm{hPa}$ averaged over the winter months (DJF) of the period $1985-90$. LMD run with no explicit orography. (a) NMC analysis; (b) LMD no drag, no lift; (c) LMD low drag only; (d) LMD low lift only. Zero line not shown; negative values are dashed.

planetary wave using SSO drag schemes, when they interact with explicit mountains. For instance, the gravity wave part of the SSO scheme improves the westerlies at the upper levels (Palmer et al. 1986) and at low levels (where the trapped waves reinforce the impact of the blocked drag), but it degrades the planetary wave over the large-scale mountains. With explicit mean orography, the lift scheme alone is beneficial for the steady planetary wave but does not affect the zonal wind very much. 

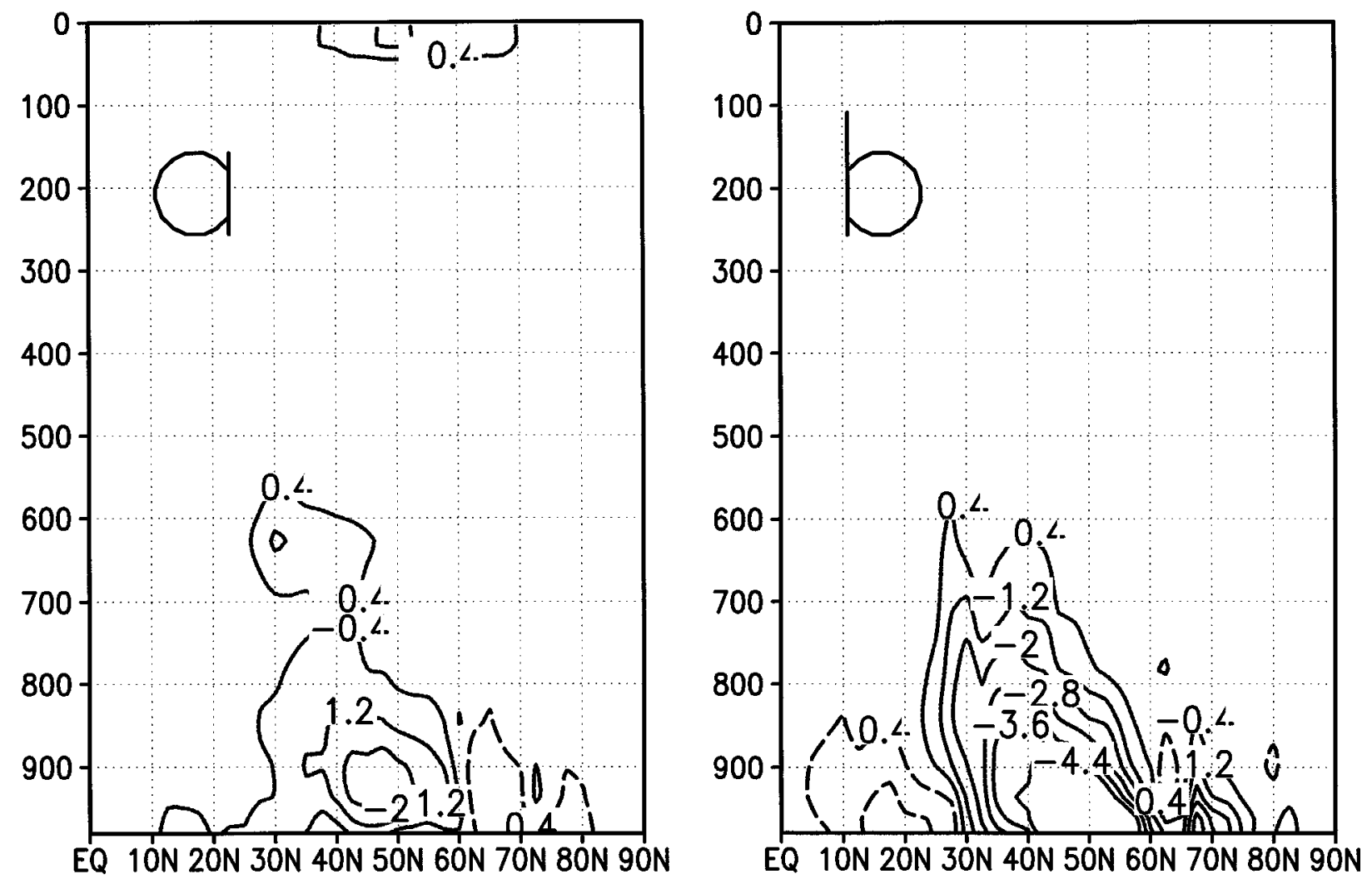

FIG. 4. Forces due to the orographic parameterization scheme, averaged over the winter months of 1985-93: zonally averaged wind tendencies. (a) Zonal wind tendency; (b) meridional wind tendency. Negative values are dashed.

With mean orography the model climate without parameterization is less in error than in the case without any mountain representation and the parameterization scheme is used to correct the model using forces that are smaller than those applied in the simulations shown in section $3 \mathrm{~b}$. In the scheme formulation itself this reduction of the parameterized forces simply follow that the blocked flow depth (1) and that the depth of the layer to which the orographic lift is applied are very much reduced when $Z_{0}=Z_{\text {mea }}$ compared to when $Z_{0}=$ 0 . In the experiment presented below, the different tunable parameters of the orographic forces scheme are $H_{\mathrm{NC}}$ $=1, C_{d}=1, G=1, \beta=0.5, \mathrm{Ri}_{c}=0.25$, and $C_{l}=$ 1 . The choice of these parameters are slightly different from that used in LM97 $\left(H_{\mathrm{NC}}=0.5, C_{d}=1, G=1\right.$, no $\left.\beta, \mathrm{Ri}_{c}=1\right),{ }^{1}$ which seems reasonable in view of the large differences between the ECMWF spectral model and the LMD GCM and also between the resolutions that were used in LM97 (T106 and T213) and that used in the present study $\left(2.5^{\circ} \times 3.5^{\circ}\right)$.

Figures $4 \mathrm{a}$ and $4 \mathrm{~b}$ show the zonally averaged orographic tendencies due to the scheme in the zonal and

\footnotetext{
${ }^{1}$ In operations ECMWF actually uses $G=0.3$ and $\mathrm{Ri}_{c}=0.25$ (M.
} Miller 1997, personal communication). in the meridional direction, respectively. The zonal tendency is essentially related to the low-level blocked flow drag and to the gravity wave drag. The combination of the blocked flow drag and of the low part (trapped lee waves) of the gravity wave drag leads to tendencies that are significant up to $600 \mathrm{hPa}$. Above the tropopause, the zonal tendency also shows a maximum that is related to the breaking of the gravity waves. The meridional tendency in Fig. 4b is essentially related to the lift parameterization and is also significant up to $600 \mathrm{hPa}$. For both the low-level drag and the low-level lift, the fact that forces are applied in the model up to such high altitudes simply follows that in some geographical areas mountain peaks can be $3000 \mathrm{~m}$ above the model-mean orography. It is also noteworthy that the intensity of lift forces in this set of experiments is larger than the intensity of the drag forces. Indeed, the parameters of the SSO scheme have been chosen to produce rather large lateral forces in order to modify significantly the steady planetary wave in the model, which is much too zonal (Fig. 1).

Further information about the orographic parameterization is also given in Figs. 5c and 5d, which show the stresses over North America related to the drag and lift parts of the scheme, respectively. It shows again that the orographic lift is larger than the orographic drag. In these 

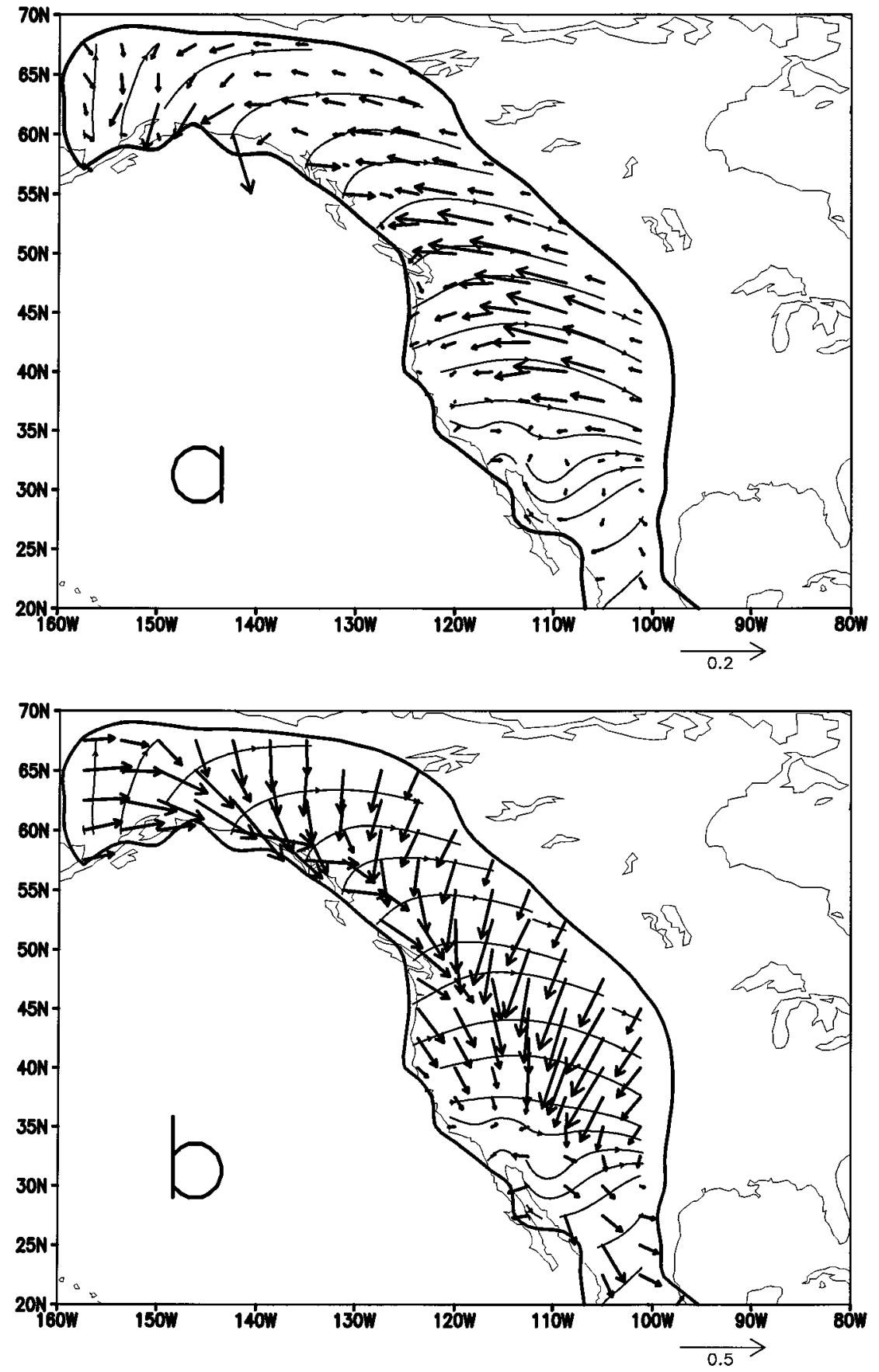

FIG. 5. Stress vectors over the Rockies due to the orographic parameterization scheme, averaged over the winter months of 1985-93; units below the arrows length scale are in Pa; thick line represents the massif limits: $Z_{\text {mea }}=1000 \mathrm{~m}$; thin line represents the streamlines of the low-level wind $\mathbf{U}_{H}$ : (a) orographic drag; (b) orographic lift.

two figures the streamfunction of the low-level wind $U_{H}$ is also represented in order to illustrate that the orographic drag parameterization gives stresses that are opposite to the low-level wind, while the lift parameterization gives stresses that are perpendicular to it.

The impact of the scheme on the model is represented on the differences map shown in Figs. 6 and 7. For both the zonal mean and the steady planetary wave it appears that the model is closer to the climatology with the orographic parameterization. The parameterized drag tends to reduce errors at nearly all levels on the zonal wind. The benefits of the scheme are nevertheless the 

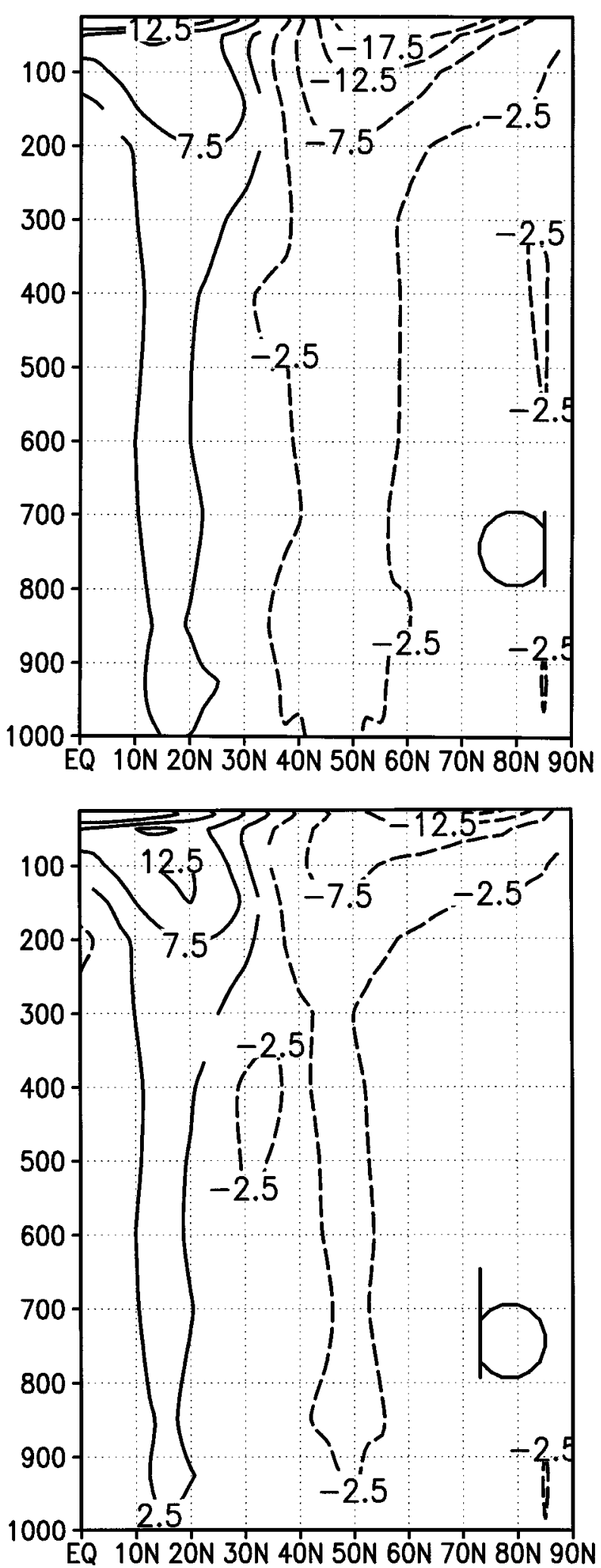

FIG. 6. Difference between the zonal wind of the NMC analysis and that of the GCM simulations: DJF 1985-93. Zero line not shown; negative values are dashed. (a) Control run; (b) run with parameterization.
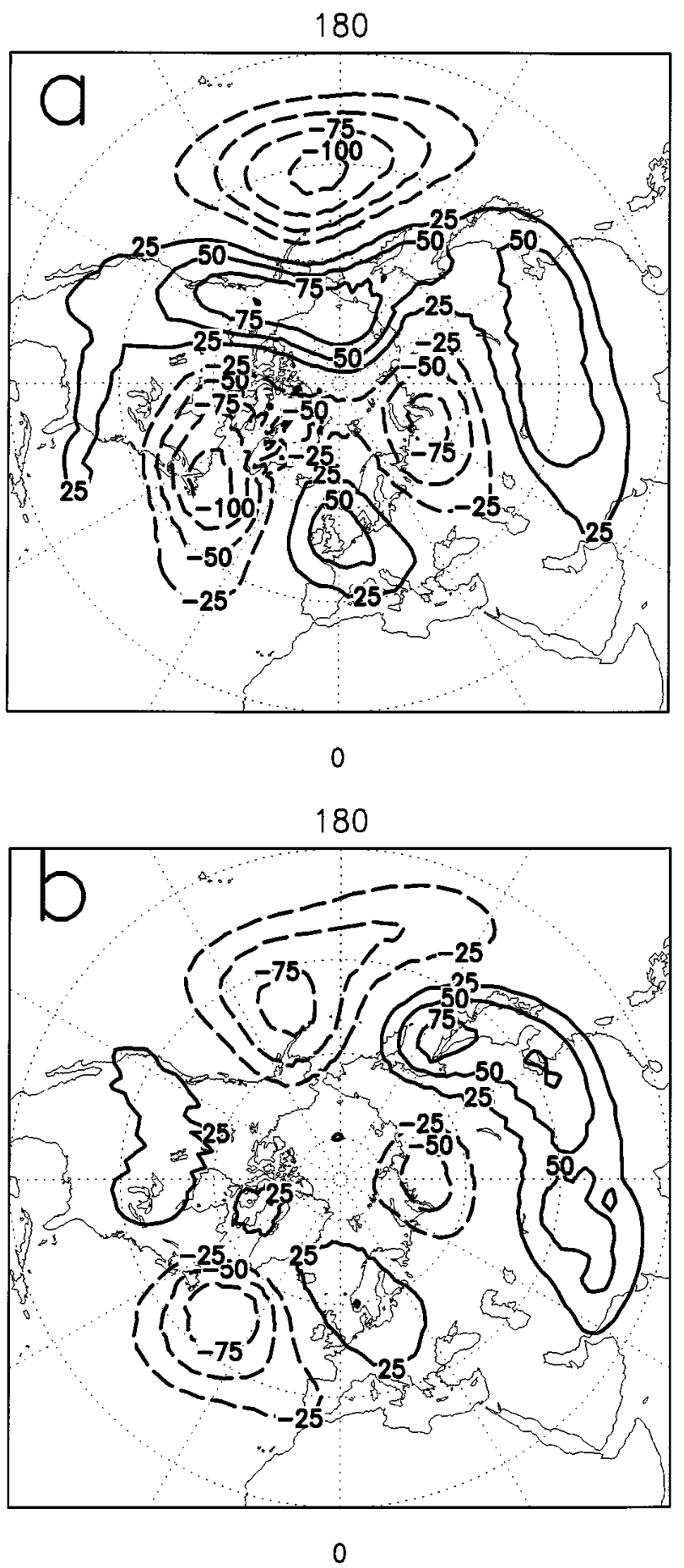

FIG. 7. Difference between the geopotential height anomaly at 500 $\mathrm{hPa}$ of the NMC analysis fields and that of the GCM simulations: DJF 1985-93. Zero line not shown; negative values are dashed. (a) Control run; (b) run with parameterization.

greatest at low levels (below $900 \mathrm{hPa}$ ) where the drag is maximum and at high levels (above $100 \mathrm{hPa}$ ) where the parameterized gravity waves break. The steady planetary wave differences are also reduced nearly every- 
where in the Northern Hemisphere, and particularly over the continents. Improvements are nevertheless more evident over North America where the scheme efficiently improves the simulation of the ridge over the Rocky Mountains. The scheme is also helpful for the simulation of the steady planetary wave over northern Europe and northern Asia, but it does not reduce the errors south and east of the Himalayan Plateau.

\section{Discussion}

On the basis of three well-known results, that is, that the cross-flow force over mountain is sometimes very large (Lott 1995), that it is associated with anticyclonic circulation over (Smith 1979) and around mountains, and that theoretical models of the steady planetary waves essentially consider orographic vortex stretching (Held 1983), it has been verified that in a GCM the reactive force to the lift exerted by the atmosphere on mountains has to be represented correctly. Indeed, numerical experiments where mountains are suppressed only recover a realistic steady planetary wave if large sideways forces are applied above the major mountain massifs. To simulate the steady planetary wave in the same context, it appears that even a highly tuned mountain drag scheme is not much help. The same experiments show that the low-level blocked part of the LM97 mountain drag schemes improves significantly the zonal-mean flow at low levels, while the lift forces does not affect it much. At first sight these results concerning mountain lift forces are not much more then a conceptual curiosity. Indeed, to try to keep the discussion as simple as possible, the sideways force representation adopted here has been taken to be linear in both the large-scale flow and the mountain volume. In this case if one assumes that the lift force is that given by Smith (1979), it only depends on the mountain volume, so that a GCM with a mean orography should represent correctly the large-scale mountains. Nevertheless, as pointed out by Wallace et al. (1983), models with mean orography sometimes understate the impact of the large-scale massifs, because mesoscale flow blocking inside the valleys causes areas of complex terrain to act on the largescale flow as if they have a larger height than that prescribed by the actual mean. In this context, and when the model lower boundary is the mean orography, the lift parameterization scheme proposed can be viewed as an SSO parameterization scheme, in the same way as the envelope orography is.

In this context, the combination of LM97's drag scheme (slightly modified to be adapted to a low-resolution GCM) and a simple enhancement of the mountain-induced lift forces improves the LMD GCM, in both the zonal-mean flow and the steady planetary wave. This shows that a recent SSO drag scheme (LM97), which was tested in a numerical weather prediction model, can also be helpful for climate models. This result is important because this scheme includes low- level forces, which are dealt with rather differently than in the past and which are often large. It means that climate models can use this type of scheme, with the advantage that they have been designed to fit the local observations better. To a certain extent, it is believed that this result is also valid for other recent orographic schemes, whose formulation follows a different approach from that used in LM97, but that were also validated against data and that also produce large drag at low levels (Shutts 1990; Broad 1996). Nevertheless, in agreement with the simulations without explicit mountains, the simulations also show that schemes that essentially decelerate the large-scale flow do not necessarily improve the steady planetary wave, and that some balance between drag and lift forces has to be considered when one tries to improve the representation of orography in GCMs.

Nevertheless, the main objective of this paper was not to propose a new parameterization of the SSO that includes realistic sideways forces. Indeed, the arguments given to justify the lift representation proposed in the context of SSO parameterization are only qualitative. The intention of the paper is rather to suggest that the direction of the forces exerted by the orography on the atmosphere should be represented properly in GCMs. Indeed, there are many examples in the literature for which the lateral forces due to mountains are important. For instance, Shutts (1995) and Broad (1995) have shown that when the wind turns with height, gravity waves encounter critical levels at nearly all altitudes and exert a drag that is perpendicular to the local flow. In these configurations, the most recent drag schemes are still not very helpful, although they begin to represent directional effects, related to the anisotropy of the mountain ridges (Phillips 1985). Other examples of "lift" can be found in oceanography, when currents encounter an island and move around it with a nonzero circulation, in a very close analogy to the classic lift exerted on a moving cylinder in an incompressible 2D inviscid flow (Batchelor 1967). Comparable effects exist in the atmosphere, when blocking occurs for certain layers that intersect mountains. As suggested by Pierrehumbert and Wyman (1985), under the action of the Coriolis force, the fluid upstream has a tendency to move around the mountain anticyclonically, with the obstacle on its right-hand side (in the Northern Hemisphere).

It is rather clear that all the results presented are probably model dependent. Indeed, the response of a GCM to a forcing is known to depend as much on the GCM formulation itself as on the forcing. For instance, if a GCM has an upper rigid lid near the tropopause and another GCM has an upper damping layer that does not reflect the planetary waves, their free modes and the associated responses to a given forcing will differ significantly. In view of this limitation, it is nevertheless believed that the example treated here shows that if there is a need to improve the representation of orographic 
forcing in a GCM (by changing the lower boundary definition or/and by some parameterization scheme), one has to consider with care the overall balance between the drag and the lift forces.

Acknowledgments. I am grateful to Dr. Martin Miller for helpful dialogues about this work. I am also grateful to Dr. Nicholas Kevlahan for a careful reading of the manuscript. Thanks also to Dr. Hervé Letreut, Dr. Robert Sadourny, and Dr. Andrew Robertson for constructive comments.

\section{APPENDIX}

\section{Modification of the Orographic Drag Scheme}

Compared to LM97, the orographic drag part of the scheme has been modified slightly, for the following reasons.

1) To have a better representation of the SSO elevation. In LM97, the envelope of the peaks is estimated from the orographic standard deviation $\mu$. It is often qualitatively correct although it leads in some places to SSO elevations that are in error. These can be easily corrected by simply using the maximum elevation calculated over the GPR.

2) To reduce the gravity wave drag when there is a blocked flow. When the atmosphere is blocked by the mountains, the forcing of the gravity waves is reduced because the effective depth of the flow passing over the ridge is lower than the total mountain height. Since this reduction strongly depends on the mountain anisotropy, an effect that is not yet well documented, it was not considered in LM97. Nevertheless, various GCM experiments have shown that in a low-resolution GCM, the gravity wave stress is very noisy and varies considerably from one grid point to another, a feature that is not expected to be resolved properly by the model. This defect is less pronounced when the "cutoff" hill concept is used because the cutoff heights are more uniform in space, all of them being quite close to $H_{\mathrm{NC}}$.

3) To represent crudely the trapped waves. There is theoretical evidence that even in the linear case some of the waves forced by the mountains remain trapped at low levels and transport momentum horizontally rather than vertically (Lott 1998). In this case, the mountain drag can still be rather well predicted by the conventional linear gravity wave drag, but the Reynolds's stress of the waves decays in the vertical direction, even in the steady undissipated case (Lott 1998).

\section{REFERENCES}

Batchelor, G. K., 1967: An Introduction to Fluid Dynamics. Cambridge University Press, $615 \mathrm{pp}$.

Bougeault, P., and Coauthors, 1993: The atmospheric momentum bud- get over a major mountain range: First results of the PYREX field program. Ann. Geophys., 11, 395-418.

Broad, A. S., 1995: Linear theory of momentum fluxes in 3-D flows with turning of the mean wind with height. Quart. J. Roy. Meteor. Soc., 121, 1891-1902.

_ 1996 : High resolution numerical model integrations to validate gravity wave drag parameterization schemes: A case study. Quart. J. Roy. Meteor. Soc., 122, 1625-1654.

Charney, J. G., and A. Eliassen, 1949: A numerical method for predicting the perturbations of the middle latitude westerlies. Tellus, 1, 38-54.

— and blocking. J. Atmos. Sci., 36, 1205-1216.

— libria and propagating planetary waves in baroclinic, orographically forced, planetary wave systems. J. Atmos. Sci., 37, 11571176.

Clark, T. L., and W. R. Peltier, 1984: Critical level reflection and the resonant growth of nonlinear mountain waves. J. Atmos. Sci., 41, 3122-3134.

D'Andrea, F., and Coauthors, 1998: Northern Hemisphere atmospheric blocking as simulated by 15 atmospheric general circulation models in the period 1979-1988. Climate Dyn., 14, 385-407.

Durran, D. R., and J. B. Klemp, 1987: Another look at downslope winds. Part II: Nonlinear amplification beneath wave-overturning layers. J. Atmos. Sci., 44, 3402-3412.

Held, I. M., 1983: Stationary and quasi-stationary eddies in the extratropical troposphere: Theory. Large-Scale Dynamical Processes in the Atmosphere, B. J. Hoskins and R. P. Pearce, Eds., Academic Press, 127-168.

Horel, J. D., and J. M. Wallace, 1981: Planetary-scale atmospheric phenomena associated with the Southern Oscillation. Mon. Wea. Rev., 109, 813-829.

Hoskins, B. J., and D. J. Karoly, 1981: The steady linear response of a spherical atmosphere to thermal and orographic forcing. $J$. Atmos. Sci., 38, 1179-1196.

- and P. J. Valdes, 1990: On the existence of storm tracks. $J$. Atmos. Sci., 47, 1855-1864.

Hunt, J., and W. H. Snyder, 1980: Experiments on stably stratified flow over a model three-dimensional hill. J. Fluid Mech., 7, 671704.

Lilly, D. K., J. M. Nicholls, R. M. Chervin, P. J. Kennedy, and J. B. Klemp, 1982: Aircraft measurements of wave momentum flux over the Colorado Rocky Mountains. Quart. J. Roy. Meteor. Soc., 108, 625-642.

Lott, F., 1995: Comparison between the orographic response of the ECMWF model and the PYREX 1990 data. Quart. J. Roy. Meteor. Soc., 121, 1323-1348.

- 1998: Linear mountain drag and averaged pseudo-momentum flux profiles in the presence of trapped lee waves. Tellus, 50A, $12-25$.

— parameterization: Its formulation and testing. Quart. J. Roy. Meteor. Soc., 123, 101-127.

Marcus, S. L., M. Ghil, and J. Dickey, 1994: The extratropical 40day oscillation in the UCLA general circulation model. Part I: Atmospheric angular momentum. J. Atmos. Sci., 51, 1431-1446.

Miller, M. J., T. N. Palmer, and R. Swinbank, 1989: Parameterization and influence of subgridscale orography in general circulation and numerical weather prediction models. Meteor. Atmos. Phys., 40, 84-109.

Miranda, P. M. A., and I. A. James, 1992: Non-linear three dimensional effects on gravity-wave drag: Splitting flow and breaking waves. Quart. J. Roy. Meteor. Soc., 118, 1057-1081.

Palmer, T. N., G. J. Shutts, and R. Swinbank, 1986: Alleviation of systematic westerly bias in general circulation and numerical weather prediction models through an orographic gravity wave drag parameterization. Quart. J. Roy. Meteor. Soc., 112, $2056-$ 2066.

Phillips, D. S., 1984: Analytical surface pressure and drag for linear 
hydrostatic flow on three-dimensional elliptical mountains. $J$. Atmos. Sci., 41, 1073-1084.

Pierrehumbert, R. T., and B. Wyman, 1985: Upstream effect of mesoscale mountains. J. Atmos. Sci., 42, 977-1003.

Queney, P., 1947: Theory of Perturbations in Stratified Currents with Application to Airflow over Mountains. Vol. 23. University of Chicago Press, 81 pp.

Sadourny, R., and K. Laval, 1984: January and July performance of the LMD general circulation model. New Perspectives in Climate Modelling, A. Berger and C. Nicolis, Eds., Elsevier, 173-198.

Schär, C., and R. B. Smith, 1993: Shallow water flow past isolated topography. Part I: Vorticity production and wake formation. $J$. Atmos. Sci., 50, 1373-1400.

— and D. R. Durran, 1997: Vortex formation and vortex shedding in continuously stratified flows past isolated topography. $J$. Atmos. Sci., 54, 534-554.

Scinocca, J., and W. R. Peltier, 1994: Finite amplitude wave activity diagnostics for Long's stationary solution. J. Atmos. Sci., 51, 613-622.

Shutts, G. J., 1990: A new gravity wave drag parameterization scheme for the unified model. TD 204, $36 \mathrm{pp}$. [Available from APR Branch, Meteorological Office, London Road, Bracknell, Berkshire RG12 252, United Kingdom.]

_ 1995: Gravity wave drag parameterization over complex terrain: The effect of critical-level absorption in directional windshear. Quart. J. Roy. Meteor. Soc., 121, 1005-1021.

Smith, R. B., 1979: Some aspects of the quasi-geostrophic flow over mountains. J. Atmos. Sci., 36, 2385-2393.

Smolarkiewicz, P., and R. Rotunno, 1989: Low Froude number flow past three-dimensional obstacles. Part I: Baroclinically generated lee vortices. J. Atmos. Sci., 46, 1154-1164.

Stephenson, D. B., 1994: The Northern Hemisphere tropospheric response to changes in the gravity-wave drag scheme in a perpetual January GCM. Quart. J. Roy. Meteor. Soc., 120, 699-712.

Valdes, P. J., and B. J. Hoskins, 1989: Linear stationary wave simulations of the time-mean climatological flow. J. Atmos. Sci., 46, 2509-2527.

Wallace, J. M., S. Tibaldi, and A. Simmons, 1983: Reduction of systematic forecast errors in the ECMWF model through the introduction of an envelope orography. Quart. J. Roy. Meteor. Soc., 109, 683-717. 